\title{
SOFTWARE DE SIMULACIÓN PARA EL DISEÑO DE REACTORES DE LODOS ACTIVADOS
}

\section{SIMULATION SOFTWARE FOR THE DESIGN OF ACTIVATED SLUDGE REACTOR}

\author{
PhD. Jairo Lenin Ramón Valencia*, PhD. Jacipt Alexander Ramón Valencia** \\ PhD. José Orlando Maldonado Bautista*** \\ * Universidad del Bosque - Programa de Bioingeniería. \\ **Universidad de Pamplona- Grupo de Investigaciones GIAAS. \\ *** Universidad de Pamplona. Grupo de Investigación CICOM. \\ E-mail: leninramon@unbosque.edu.co, \{jacipt, orlmaldonado\}@unipamplona.edu.co
}

\begin{abstract}
Resumen: El presente artículo describe el diseño y desarrollo de una herramienta computacional, que permite modelar un sistema de tratamiento de aguas residuales urbanas por medio de un reactor biológico de lodos activados. El sistema de lodos activados se basa en tres diseños: mezcla completa (reactor sin recirculación y reactor con recirculación) y flujo pistón con recirculación. La información obtenida sirve como base de diseño para la selección de un sistema de manejo integral de aguas a partir de lodos activados. La implementación del modelo, y su posterior simulación mediante el software sirve para diseñar o modelar plantas de tratamiento de aguas residuales, o como herramienta de apoyo para las tareas de supervisión, permitiendo conocer los valores óptimos con los que debe trabajar su sistema de tal forma que se pueda tener una adecuada interpretación de los resultados.
\end{abstract}

Palabras clave: Reactor biológico, lodos activados, modelo computacional, aireación, mezcla completa, flujo pistón con recirculación.

\begin{abstract}
In this research it was designed and developed a computational tool, which models a system of treatment of urban waste water by Means of an activated sludge biological reactor. The activated sludge system is based on three designs: Complete mixture (reactor without recirculation and recycle reactor) and plug flow recirculation. The information obtained serves as a design basis for the selection of a system of integrated management of water from activated sludge. The implementation of the model and its mathematical simulation can be used to design or model plants to sewage treatment. It can also be useful for who oversees a plant and want to know the optimum values which should work with your system and facilitate interpretation of results.
\end{abstract}

Keywords: Bioreactor, activated sludge treatment, computing model, aeration, complete mix, plug flow recirculation.

\section{INTRODUCCIÓN}

El proceso de lodos activados se desarrolla en Inglaterra; todos los procesos de lodos activados tienen en común el contacto de aguas residuales con un floc biológico previamente formado en un tanque de aireación; el lodo activado consiste en una masa floculante de microorganismos, materia orgánica muerta y materiales inorgánicos; tiene la propiedad de poseer una superficie altamente activa para la adsorción de materiales coloidales o 
suspendidos, a la cual debe su nombre de activado. El resultado final es una porción de materia orgánica, apta de descomposición biológica, convertida en compuestos inorgánicos y el resto, transformada en lodo activo adicional (Romero, Jairo Alberto. 2008). Un sistema convencional de lodos activados consta de un tanque de aireación, sedimentador y recirculación de los lodos activados, donde:

Tanque de aireación: tiene un tamaño fijo y por consiguiente un tiempo de retención fijo para un caudal determinado, el tamaño del tanque debe ser suficiente para permitir que los microorganismos alcancen la fase endógena durante los periodos de caudal máximo y máxima carga orgánica; siendo este el más importante en el proceso de lodos activados

Existen varios Sistemas Convencionales para Lodos Activados como:

- Sistema convencional con aireación gradual

- Sistema convencional con aireación prolongada

- Proceso de mezcla completa

- Proceso de Kraus

- Proceso de tasa alta

- Proceso de estabilización y contacto

- Proceso de aireación prolongada

Para este estudio resaltaremos el Sistema convencional con aireación gradual (Reactor con flujo pistón) y el Proceso de mezcla completa (Reactor con recirculación y Reactor sin recirculación), por consiguiente se hizo una programación de ecuaciones características para cada uno de estos tres procesos biológicos con ayuda de Matlab.

\section{METODOLOGÍA}

Cada uno de estos sistemas de tratamiento biológico de flujo continuo tiene características específicas de funcionamiento.

\subsection{Sistema convencional con aireación gradual}

El tanque de aireación es largo y rectangular, con difusores a lo largo de una pared, para oxigenación y mezcla en espiral. El suministro de aire es gradual, a lo largo del tanque para promover máxima aireación a la entrada por donde ingresa el afluente y los lodos recirculados (RAS. 2005).

\subsubsection{Modelo en flujo pistón con recirculación:}

En este sistema se supone que existe mezcla completa en sección transversal (horizontal) de flujo, sin ninguna mezcla en la dirección longitudinal, sus expresiones cinéticas cuantificables ya fueron deducidas por lo tanto se supone que la concentración de biomasa $(\mathrm{X})$ en el reactor es constante. La fig. 1 ilustra este fenómeno.

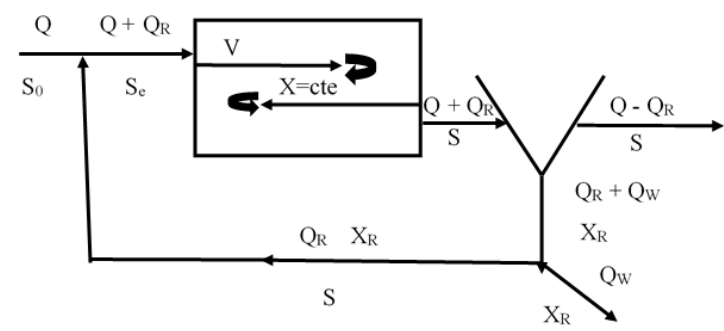

Fig. 1. Flujo en pistón con recirculación

$\mathrm{Q}=$ caudal

$\mathrm{Q}_{\mathrm{R}}=$ caudal de recirculación

$\mathrm{Q}_{\mathrm{w}}=$ caudal de desecho

$\mathrm{V}=$ volumen reactor

$\mathrm{S}=$ concentración de DQO

$\mathrm{X}=$ concentración de biomasa en el reactor

$\mathrm{X}_{\mathrm{R}}=$ concentración de biomasa de recirculación

\subsection{Proceso de mezcla completa}

Se provee mezcla completa y continua, mediante aireación mecánica o por difusores donde se busca disminuir los desequilibrios biológicos, causados por amplias variaciones horarias en la carga orgánica o hidráulica, en efluentes de pequeñas comunidades (Orozco, A. 2007 y 2014).

\subsubsection{Modelo de Mezcla Completa sin Recirculación}

$\mathrm{Q}=$ caudal

$\mathrm{V}=$ volumen reactor

$\mathrm{S}_{\mathrm{o}}=$ concentración de DQO de entrada o afluente

$\mathrm{X}_{\mathrm{o}}=$ concentración de biomasa de entrada (afluente)

$\mathrm{X}=$ concentración de biomasa en el reactor

$\mathrm{S}=$ concentración de DQO en el reactor

En la figura 2, se puede observar dicho modelo.

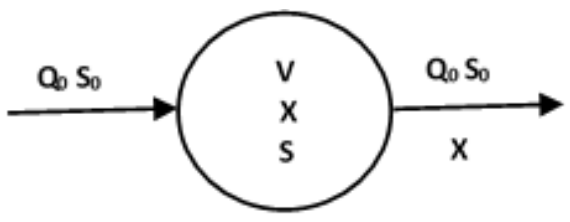

Mezcla completa sin recirculación

Fig. 2. Modelo mezcla completa sin recirculación 


\subsubsection{Modelo de Mezcla Completa con Recirculación}

En este sistema se supone que el contenido del reactor está mezclado completamente, todas las reacciones ocurren en el tanque de aireación y que la concentración de biomasa (X) en el afluente es despreciable. La figura 3 esquematiza dicho modelo.

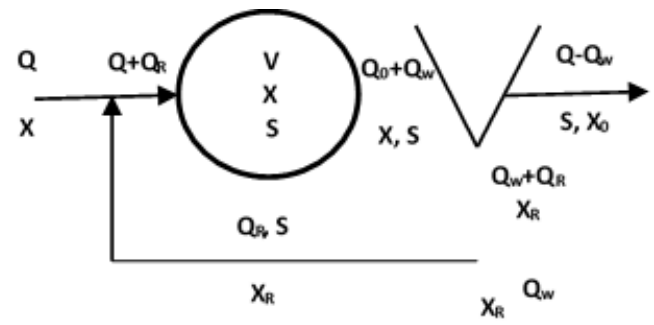

Mezcla completa con recirculación

Fig. 3. Modelo de mezcla completa con recirculación

$\mathrm{Q}=$ caudal

$\mathrm{Q}_{\mathrm{R}}=$ caudal de recirculación

$\mathrm{Q}_{\mathrm{w}}=$ caudal de desecho

$\mathrm{V}=$ volumen reactor

$\mathrm{S}_{\mathrm{O}}=$ concentración de DQO en el afluente

$\mathrm{S}=$ concentración de DQO en el reactor

$\mathrm{X}=$ concentración de biomasa en el reactor

$\mathrm{X}_{\mathrm{R}}=$ concentración de biomasa de recirculación

En el preliminar se exhibió como es el mecanismo de cada uno de los reactores y que sucede en cada uno de sus movimientos.

\section{RESULTADOS}

Teniendo en cuenta las características anteriormente se decide implementar un software como herramienta que permita el cálculo de diseños de reactores biológicos de lodos activados a partir de diferentes modelos (mezcla completa sin recirculación-mezcla completa con recirculaciónflujo pistón con recirculación), implementando una interfaz gráfica para mejorar su usabilidad.

Dentro de los objetivos finales del proyecto están el desarrollo de una aplicación standalone, desarrollada en lo posible con herramientas de software libre, sin problemas de licenciamiento y que permita que su instalación y ejecución sea independiente de la plataforma, así como el código abierto para su modificación, mejoramiento y escalabilidad (Martínez, S. Rodríguez M. 2005).
Tabla 1. Ecuaciones para procesos biológicos con crecimientos suspendidos

\begin{tabular}{|c|c|}
\hline Parametro & Flujo piston con Recirculacion \\
\hline Eficencia & $E=\frac{S_{0}-S}{S_{0}}$ \\
\hline $\begin{array}{c}\text { Concentracion } \\
\text { de efluente }\end{array}$ & $\mathrm{X}=\frac{Y\left(s_{0} * s\right) \theta_{c}}{\left(1+K_{d} * \theta_{c}\right) \theta}$ \\
\hline $\begin{array}{c}\text { Biomasa en el } \\
\text { Reactor }\end{array}$ & $P_{x}=\frac{Y Q\left(s_{0} * s\right)}{1+K_{d} * \theta_{c}}$ \\
\hline $\begin{array}{c}\text { Produccion de } \\
\text { Lodos }\end{array}$ & $\theta=\theta_{c}\left(1+\mathrm{R}-\mathrm{R} \frac{X_{R}}{X}\right)$ \\
\hline $\begin{array}{c}\text { Tiempo de } \\
\text { Retencion de } \\
\text { Lodos (V/Q) }\end{array}$ & $\frac{1}{\theta_{c}}=\frac{Y k S}{K_{S} \ln \left(\frac{S_{0}}{S}\right)+\left(S_{0}-S\right)}-K_{d}$ \\
\hline $\begin{array}{c}\text { Edad de Lodos } \\
\text { Edad Minima } \\
\text { de Lodos }\end{array}$ & - \\
\hline
\end{tabular}

Tabla 2. Otras Ecuaciones para procesos biológicos con crecimientos suspendidos

\begin{tabular}{|c|c|c|}
\hline \multicolumn{3}{|c|}{ Mezcla Completa } \\
\hline Parametro & Sin recirculacion & Con Recirculacion \\
\hline $\begin{array}{c}\text { Concentracion } \\
\text { de efluente }\end{array}$ & $S=\frac{K_{S}\left(1+K_{d} * \theta_{c}\right)}{S_{0}}$ & $E=\frac{S_{0}-S}{\theta_{0}}$ \\
\hline $\begin{array}{c}\text { Biomasa en el } \\
\text { Reactor }\end{array}$ & $\mathrm{X}=\frac{Y\left(s_{0} * S\right)}{1+K_{d} * \theta_{c}}$ & $\mathrm{X}=\frac{Y\left(S_{0}\left(1+K_{d} * \theta_{c}\right)\right.}{\theta_{c}\left(Y k-K_{d}\right)-1}$ \\
\hline $\begin{array}{c}\text { Produccion de } \\
\text { Lodos }\end{array}$ & $P_{x}=\frac{Y Q\left(s_{0} * S\right)}{1+K_{d} * \theta_{c}}$ & $P_{x}=\frac{Y Q\left(s_{0} * S\right)}{1+K_{d} * \theta_{c}}$ \\
\hline $\begin{array}{c}\text { Tiempo de } \\
\text { Retencion de } \\
\text { Lodos (V/Q) }\end{array}$ & $\theta=\theta_{c}$ & $\theta=\theta_{c}\left(1+\mathrm{R}-\mathrm{R} \frac{X_{R}}{X}\right)$ \\
\hline $\begin{array}{c}\text { Edad de Lodos } \\
\text { Edad Minima } \\
\text { de Lodos }\end{array}$ & $\frac{1}{\theta_{c}}=\frac{Y k S}{K_{s}+S}-\frac{Y k S_{0}}{K_{S}+S_{0}}-K_{d}$ & $\frac{1}{\theta_{c}}=\frac{Y k S}{K_{S}+S}-K_{d}$ \\
\hline
\end{tabular}

Con estas características se desarrolla el programa que cuenta con las siguientes características:

La primera parte inicia con una presentación del programa en la que se presenta un esquema del fenómeno modelado y se dan algunas explicaciones generales, esto como se muestra en la figura 4. 


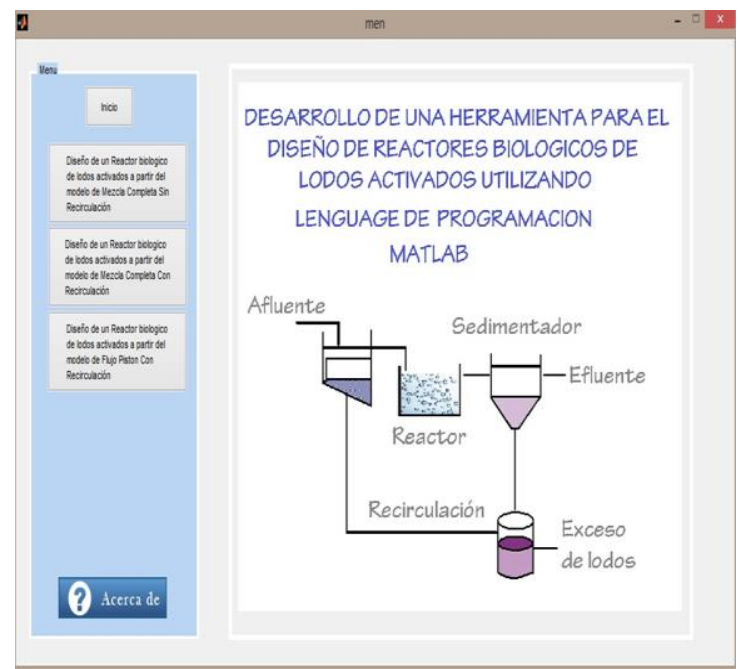

Fig. 4. Inicio del programa

En una segunda ventana se realizan los cálculos correspondientes al modelo de mezcla completa sin recirculación, lo cual es similar para los otros modelos, En dicha ventana se ofrecen los cuadros de texto donde hay que introducir los datos de entrada para seguidamente calcular los datos de salida, aplicando las fórmulas del modelo.

La figura 5 muestra la interfaz para que el usuario introduzca los valores de entrada y se puedan realizar los cálculos. También se describen los datos de entrada y salida para facilitar el trabajo del usuario.

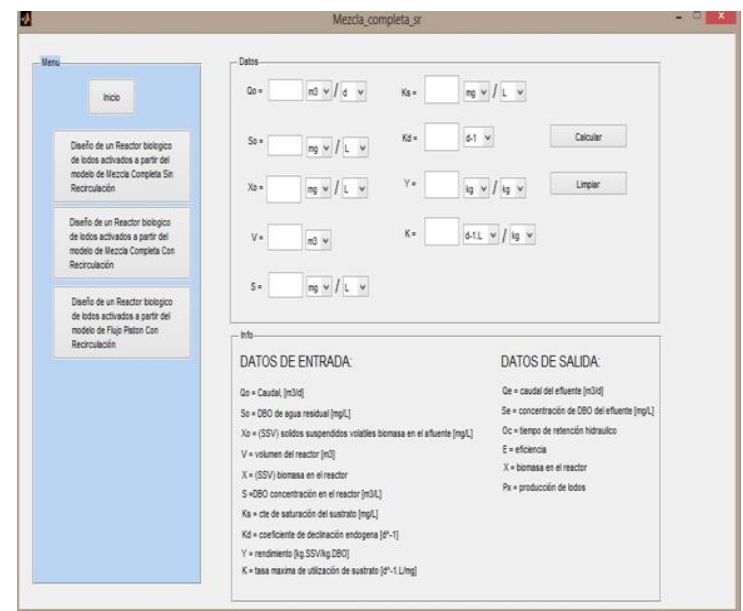

Fig. 5. Interfaz para entrada de datos.

Al pulsar el botón calcular, inmediatamente se muestra un panel de salida con los resultados esperados, de acuerdo a los valores ingresados, tal como se muestra en la figura 6.

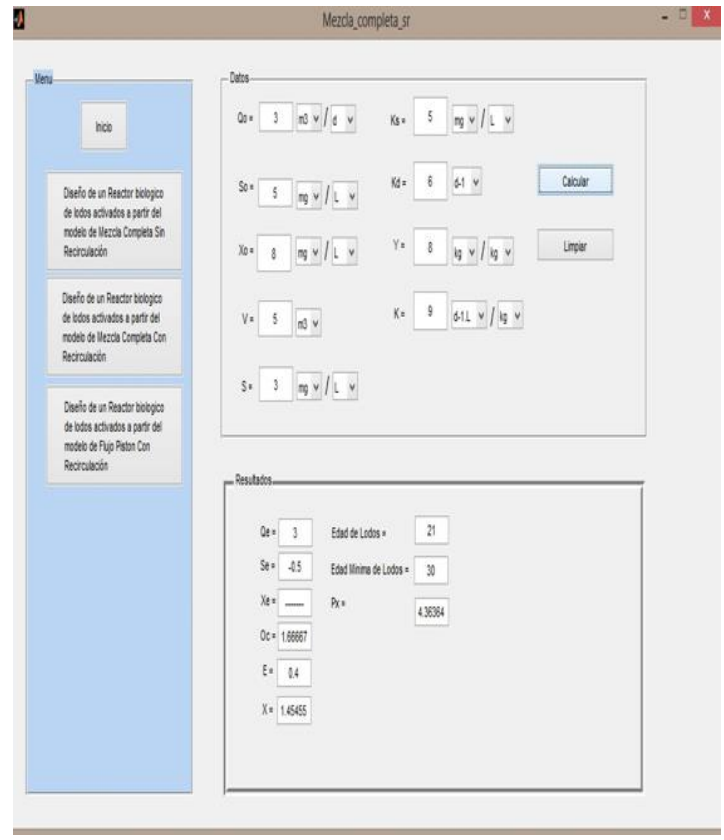

Fig. 6. Panel con salida de los resultados

Para evitar el ingreso de datos erróneos, el software tiene un sistema de validación que advierte al usuario que debe revisar sus entradas, tal como se aprecia en la figura 7 .
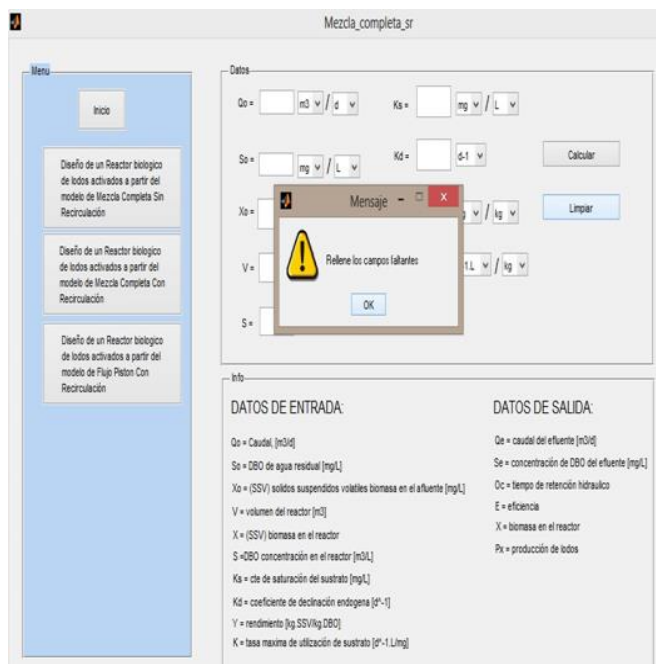

Fig. 7. Sistema de validación de datos erróneos

Para cada tipo de reactor se hizo una modelación diferente, por lo tanto, al inicio del programa se escoge con que reactor se desea trabajar por consiguiente nosotros ingresamos valores necesarios para que se ejecute y nos caracterice lo propuesto. 


\section{CONCLUSIONES}

Se ha desarrollado una herramienta computacional que permite el modelado de un sistema de tratamiento de aguas residuales urbanas por medio de un reactor biológico de lodos activados.

La herramienta implementa las ecuaciones del modelo y permite centrarse en la simulación mediante el ingreso de los posibles datos de entrada.

El prototipo muestra el potencial que tiene como herramienta tanto para el uso técnico y científico, como software educativo para utilización en el aula.

Una segunda etapa del proyecto consiste, por un lado en el refinamiento del modelo, por otro en la utilización de software libre para desarrollar una aplicación de código abierto, libre distribución y libre uso, y finalmente, se espera poder simular el proceso de forma que se aprecie el fenómeno físico, además de los resultados numéricos.

\section{REFERENCIAS}

Romero Rojas, Jairo Alberto (2004). Tratamiento de aguas residuales. Teoría y principios de diseño. Editorial: Escuela Colombiana de Ingeniería. Tercera edición. $1248 \mathrm{p}$.

Martínez, S. Rodríguez M. (2005). Tratamiento de aguas residuales con Matlab $^{\circledR}$. Universidad Autónoma Metropolitana, México: Editorial Reverté S. A.

Orozco, A. (2007). Teoría de la remoción de sustrato soluble en plantas de lodos activados Bogotá: Revista Acodal.

Orozco, A. (2014). Bioingeniería de aguas residuales, Segunda edición, Bogotá: Editorial Acodal.

RAS. (2005). Reglamento técnico del sector de agua potable y saneamiento básico Título $\mathrm{E}$. Minvivienda, Colombia.

Romero, Jairo Alberto. (2008). Tratamiento de aguas residuales teoría y principios de diseño. Bogotá: Editorial Escuela Colombiana de Ingeniería. 